\title{
Prognostic factors for survival of patients with nasopharyngeal carcinoma following conventional fractionation radiotherapy
}

\author{
FEI KONG, BAI-ZHEN CAI, XIAN-ZHAO CHEN, JIAN ZHANG and YI-MING WANG \\ Department of Radiotherapy, The People's Hospital of Hainan Province, Haikou, Hainan 570311, P.R. China
}

Received December 7, 2012; Accepted February 8, 2013

DOI: $10.3892 /$ etm.2013.1100

\begin{abstract}
This study aimed to investigate the risk factors influencing the prognosis of patients receiving conventional fractionation radiotherapy. A retrospective analysis of clinical data from 100 patients with nasopharyngeal carcinoma receiving radiotherapy was conducted. The Chi-square test was used to screen the relevant factors and Cox multiple-factor analysis was used to investigate the risk factors influencing the survival of patients. One-factor analysis results revealed that tumor stage, tumor diameter, prescription dose completion and radiotherapy regularity are related to the prognosis of nasopharyngeal carcinoma and multiple-factor analysis results revealed that tumor stage, radiotherapy dose and radiotherapy regularity are independent risk factors influencing prognosis. The prognosis of patients with nasopharyngeal carcinoma receiving radiotherapy is related to tumor progression and an adequate dose of regular radiotherapy improves the prognosis of patients.
\end{abstract}

\section{Introduction}

Nasopharyngeal carcinoma is the most common malignant tumor in the head and neck region and $80 \%$ of nasopharyngeal carcinoma cases worldwide occur in China. The incidence of nasopharyngeal carcinoma significantly increases in individuals aged $>30$ years and $80 \%$ of cases occur in individuals aged 30-60 years (1-3). Nasopharyngeal carcinoma is sensitive to radiotherapy and the short-term treatment efficacy is good; however, the recurrent rate and the distant metastasis rate remain high. Therefore, the prognosis varies (4-7). Clinical studies have confirmed that among patients with nasopharyngeal carcinoma receiving radiotherapy, tumor progression is rapid and clinical prognosis is poor, even if patients begin radiotherapy immediately $(8,9)$. We conducted a retrospective

Correspondence to: Dr Fei Kong, Department of Radiotherapy, The People's Hospital of Hainan Province, XiuHua Road 19, Haikou 570311, P.R. China

E-mail:kfymcn@yeah.net

Key words: nasopharyngeal carcinoma, conventional fractionation radiotherapy, prescription dose, tumor stage analysis of clinical data from patients with nasopharyngeal carcinoma receiving radiotherapy in our hospital to investigate the relevant factors influencing the prognosis and survival of patients, as well as the independent risk factors, to provide a reference for the evaluation of nasopharyngeal carcinoma radiotherapy and prognosis.

\section{Materials and methods}

Clinical data. A total of 100 patients with nasopharyngeal carcinoma who received radiotherapy in the Radiotherapy Department in our hospital from January 2006 to December 2006 were selected. There were 62 male and 38 female cases, aged $18-78$ years. The mean age was $51 \pm 18.6$ years. Diagnoses of all patients were confirmed by pathological examination. According to the standard of nasopharyngeal carcinoma diagnosis and treatment prepared by the Otolaryngology Branch of Chinese Medical Association (in 1992, Fuzhou, China), there were 91 cases in stage I, 24 cases in stage II, 36 cases in stage III and 21 cases in stage IV. Among them, there was 1 case of carcinoma sarcomatodes and 99 cases of poorlydifferentiated squamous cell carcinoma. Additionally, there were 68 cases with completed prescription dose radiotherapy, 27 cases with severe radiotherapy complications and 65 cases that received regular radiotherapy. This study was approved by the ethics committee of People's Hospital of Hainan Province (Haikou, China). The informed consent was obtained from all patients.

Radiotherapy. Prior to therapy, computed tomography (CT), magnetic resonance imaging (MRI) and electroconvulsive therapy (ECT) examinations were performed for all patients in order to evaluate the foci. With the exception of patients with lesion metastases, all other patients received targeted conventional fractionation radiotherapy using a Varian 23EX-518 medical linear accelerator (6 MV $\mathrm{X}$-ray and $9 \mathrm{MeV} \beta$-ray). The primary clinical target area encompassed the gross tumor volume (GTV) plus a 5-10-mm extension, and the lymphatic drainage area encompassed the GTV plus a 3-mm extension. The outlined target area data were processed by the treatment planning system (TPS) to prepare the radiotherapy plan and prescription dose. The radiotherapy dose for the primary foci and cervical lymph node metastasis foci was 60-70 Gy and the prophylactic neck dose was $50 \mathrm{~Gy}$, fractionated 30 times for irradiation. The 
Table I. Analysis of factors related to the prognosis of patients with nasopharyngeal carcinoma radiotherapy.

\begin{tabular}{|c|c|c|c|c|c|}
\hline \multirow[b]{2}{*}{ Factors } & \multirow[b]{2}{*}{$\mathrm{n}$} & \multicolumn{3}{|c|}{ No. of survivors } & \multirow[b]{2}{*}{ P-value } \\
\hline & & 1 year & 3 years & 5 years & \\
\hline \multicolumn{6}{|l|}{ Gender } \\
\hline Female & 38 & 37 & 35 & 32 & \multirow[t]{2}{*}{$>0.05$} \\
\hline Male & 62 & 56 & 45 & 41 & \\
\hline \multicolumn{6}{|c|}{ Age (years) } \\
\hline$\geq 51$ & 44 & 37 & 36 & 34 & \multirow[t]{2}{*}{$>0.05$} \\
\hline$<51$ & 56 & 56 & 50 & 39 & \\
\hline \multicolumn{6}{|c|}{ Tumor diameter $(\mathrm{cm})$} \\
\hline$<2.4$ & 42 & 41 & 38 & 35 & \multirow[t]{2}{*}{$<0.05$} \\
\hline$\geq 2.4$ & 58 & 47 & 43 & 38 & \\
\hline \multicolumn{6}{|c|}{ Cancer stage } \\
\hline I & 19 & 19 & 16 & 16 & \multirow[t]{4}{*}{$<0.05$} \\
\hline II & 24 & 24 & 22 & 22 & \\
\hline III & 36 & 35 & 30 & 30 & \\
\hline IV & 21 & 15 & 13 & 5 & \\
\hline \multicolumn{6}{|c|}{ Prescription dose completed } \\
\hline Yes & 68 & 66 & 63 & 62 & \multirow[t]{2}{*}{$<0.05$} \\
\hline No & 32 & 27 & 18 & 11 & \\
\hline \multicolumn{6}{|c|}{ Regular radiation } \\
\hline Yes & 65 & 64 & 60 & 57 & \multirow[t]{2}{*}{$<0.05$} \\
\hline No & 35 & 29 & 21 & 16 & \\
\hline \multicolumn{6}{|c|}{ Serious radiotherapy complications } \\
\hline Yes & 27 & 24 & 19 & 14 & \multirow[t]{2}{*}{$>0.05$} \\
\hline No & 73 & 69 & 62 & 59 & \\
\hline
\end{tabular}

non-target area and sensitive organs were shaded and the received dose was no more than the tolerance dose. In addition, CT scanning was conducted every 2 weeks to evaluate treatment efficacy, and the radiotherapy range and ray dose were determined again.

Factor analysis and assignment method. The clinical data of patients were sorted and classified. Data for analysis included gender (male compared with female), age (patients over the mean age compared with patients under the mean age), tumor stage, tumor diameter (tumors with diameter greater than the mean compared with those with diameter less than the mean), radiotherapy dose (patients who completed the prescription dose compared with patients who did not), radiotherapy regularity (patients who received regular compared with irregular radiotherapy) and radiotherapy complications (patients experiencing complications compared with those with no complications). Survival time was recorded according to the follow-up records for patients. The cases lost to follow-up were excluded.

Statistical analysis. Data were tabled using Excel and processed using the Chinese version of SPSS 17.0 statistical software. Survival time was analyzed by the Kaplan-Meier test, the relevant factors were screened by chi-square test and independent risk factors were analyzed by the Cox proportional hazards model. $\alpha=0.05$ was considered to indicate a statistically significant difference.

\section{Results}

Survival time and survival rate. Kaplan-Meier survival analysis results revealed that the median survival time of patients with nasopharyngeal carcinoma receiving radiotherapy was 52 months. The 1-, 3- and 5-year survival rates were 93.0, 80.0 and $73.0 \%$, respectively.

Analysis of prognosis-related factors. Among the 100 patients with nasopharyngeal carcinoma receiving radiotherapy, there were 62 male and 38 female cases. Of these, 56 cases were $<51$ years old and 44 cases were $\geq 51$ years old. The tumor diameter was $<2.4 \mathrm{~cm}$ in 42 cases and $\geq 2.4 \mathrm{~cm}$ in 58 cases. Furthermore, 19 cases were stage I, 24 cases were stage II, 36 cases were stage III and 21 cases were stage IV. In addition, 68 cases completed prescription dose radiotherapy, 65 cases received regular radiotherapy and 27 cases presented severe radiotherapy complications. The results of one-factor analysis revealed that gender, age and severe radiotherapy complication of patients were not significantly associated with the prognosis of patients (all $\mathrm{P}>0.05$ ), while tumor stage, tumor diameter, 
Table II. Analysis of independent risk factors for survival of patients receiving radiotherapy for nasopharyngeal carcinoma.

\begin{tabular}{lcccccc}
\hline Factors & $\beta$-value & Standard error & Wald value & OR value & $95 \%$ CI & P-value \\
\hline Cancer stage & 0.675 & 0.214 & 10.140 & 1.796 & $0.599-3.106$ & 0.004 \\
$\begin{array}{l}\text { Prescription dose } \\
\text { radiotherapy }\end{array}$ & -0.724 & 0.269 & 7.926 & 0.692 & $1.412-2.969$ & 0.003 \\
Rule radiation & -0.892 & 0.304 & 7.108 & 1.549 & $0.826-2.742$ & 0.002 \\
\hline
\end{tabular}

OR, odds ratio; CI, confidence interval.

prescription dose completion and radiotherapy regularity were significantly associated with the prognosis of patients (all $\mathrm{P}<0.05)$. The results are presented in Table I. We identified that a tumor diameter $\geq 2.4 \mathrm{~cm}$, an advanced stage, uncompleted prescription dose and regular radiotherapy are factors related to a poor prognosis of nasopharyngeal carcinoma radiotherapy.

Risk factors. According to one-factor analysis, we identified that four factors affect prognosis. Therefore, a logistic model was induced. Cox multiple-factor analysis results revealed that nasopharyngeal carcinoma tumor stage, prescription dose completion and radiotherapy regularity were independent risk factors influencing the survival of patients. Advanced tumor stages increase the risk of mortality $(\mathrm{P}=0.004)$. In addition, completed prescription dose $(\mathrm{P}=0.003)$ and regular radiotherapy $(\mathrm{P}=0.002)$ significantly reduce the risk of mortality. The results are presented in Table II.

\section{Discussion}

There are numerous nasopharyngeal structures and nasopharyngeal carcinoma is the most common malignant tumor in the nasopharynx. Radiotherapy is the main treatment method for nasopharyngeal carcinoma. For the majority of nasopharyngeal carcinomas, radiotherapy improves the clinical result. Clinical studies have confirmed that following radiotherapy in patients with nasopharyngeal carcinoma, the 5-year survival rate reaches $50-80 \%$ (10-12). Although radiotherapy produces an ideal result for the majority of nasopharyngeal carcinomas, the median survival time for patients with nasopharyngeal carcinoma receiving radiotherapy in this study was 52 months and the 5-year survival rate was $73.0 \%$, consistent with previous results (13-15). Despite the improved clinical efficacy observed in a number of cases of nasopharyngeal carcinoma, tumor recurrence and metastasis are the main causes of treatment failure.

Among certain individuals, prognoses of radiotherapy are inconsistent. Therefore, it is important to evaluate the prognosis of patients prior to radiotherapy. One clinical study (16) confirmed that the clinical stage of nasopharyngeal carcinoma is closely related to the prognosis. Furthermore, the tumorous metastasis stage is an independent risk factor influencing prognosis and is also the main influence on shortterm efficacy for nasopharyngeal carcinoma. According to the data in the present study, the prognosis of patients with nasopharyngeal carcinoma receiving radiotherapy is not associated with age, gender or radiotherapy complications; however, tumor stage, tumor diameter, prescription dose completion and radiotherapy regularity are associated with prognosis.

In nasopharyngeal carcinoma, the onset age range is larger and young patients are common. However, we did not identify an association between age and prognosis. The main cause of nasopharyngeal carcinoma onset is nasopharyngeal viral infection; however, previous studies did not identify a correlation between nasopharyngeal carcinoma and sex hormones. Additionally, tumor diameter and stage of nasopharyngeal carcinoma are related to prognosis, suggesting that tumorous biological behavior remains one of the main influences on the prognosis of patients with nasopharyngeal carcinoma receiving radiotherapy $(10,17,18)$.

For nasopharyngeal carcinoma with a large volume and an advanced stage, the possibility of tumorous residues following radiotherapy is greater and these are the main causes of tumor recurrence following treatment. If a poorly differentiated nasopharyngeal carcinoma is recurrent, its highly malignant biological behavior usually causes rapid progression of the tumor, resulting in a poor prognosis. Among radiotherapyrelated factors, prescription dose completion and radiotherapy regularity are related to prognosis, while radiotherapy complications are not related to prognosis. The common complications of radiotherapy for the treatment of nasopharyngeal carcinoma include adjacent neural injury, radiation dermatitis of the head and neck and oral mucosa injury. These often affect the quality of life of patients.

With the development of studies highlighting radiotherapy, the possibility of fatal injury to the spinal cord and other important organs is lower. Therefore, if patients complete the radiotherapy plan, there are fewer influences on prognosis. In the treatment of nasopharyngeal carcinoma with radiotherapy, completed planned prescription dose and regular radiotherapy are vital $(19,20)$. The prescription dose is the load dose required to remove tumor tissues and cells in the target area to the maximum extent. Regular radiotherapy achieves the expected efficacy of the prescription dose.

The results in this study demonstrate that the malignant biological behavior, progression and radiotherapy implementation according to the plan of nasopharyngeal carcinoma are the factors influencing the prognosis of patients with nasopharyngeal carcinoma receiving radiotherapy. Therefore, for patients with nasopharyngeal carcinoma receiving radiotherapy, it is necessary to accurately evaluate tumor progression and strictly implement radiotherapy according to the plan to achieve an improved clinical efficacy. 


\section{Acknowledgements}

This study was supported by the Natural Science Foundation of Hainan Province, China (No. 805102).

\section{References}

1. Adham M, Kurniawan AN, Muhtadi AI, et al: Nasopharyngeal carcinoma in Indonesia: epidemiology, incidence, signs and symptoms at presentation. Chin J Cancer 31: 185-196, 2012.

2. Huang TR, Zhang SW, Chen WQ, et al: Trends in nasopharyngeal carcinoma mortality in China, 1973-2005. Asian Pac J Cancer Prev 13: 2495-2502, 2012.

3. Li JX, Lu TX, Huang Y and Han F: Clinical characteristics of recurrent nasopharyngeal carcinoma in high-incidence area. Scientific World Journal: Feb 1, 2012 (Epub ahead of print).

4. Teo P, Yu P, Lee WY, et al: Significant prognosticators after primary radiotherapy in 903 nondisseminated nasopharyngeal carcinoma evaluated by computer tomography. Int J Radiat Oncol Biol Phys 36: 291-304, 1996.

5. Geara FB, Sanguineti G, Tucker SL, et al: Carcinoma of the nasopharynx treated by radiotherapy alone: determinants of distant metastasis and survival. Radiother Oncol 43: 53-61, 1997.

6. Sanguineti G, Geara FB, Garden AS, et al: Carcinoma of the nasopharynx treated by radiotherapy alone: determinants of local and regional control. Int J Radiat Oncol Biol Phys 37: 985-996, 1997.

7. Erkal HS, Serin M and Cakmak A: Nasopharyngeal carcinomas: analysis of patient, tumor and treatment characteristics determining outcome. Radiother Oncol 61: 247-256, 2001.

8. Zhao C, Lu TX, Han F, et al: Clinical study of 139 nasopharyngeal carcinoma patients for intensity modulated radiation therapy. Chin J Radiat Oncol 15: 1-6, 2006.

9. Tham IW and Lu JJ: Controversies and challenges in the current management of nasopharyngeal cancer. Expert Rev Anticancer Ther 10: 1439-1450, 2010.
10. Tombolini V,De Sanctis V, Donato V, et al: Prognostic features and treatment outcome in patients with nasopharyngeal carcinoma: an experience of 20 years. Anticancer Res 21: 1413-1418, 2001.

11. Wang XS, Hu CS, Wu YR and Feng Y: Influence of speed in tumor regression on prognosis of nasopharyngeal carcinoma. Chin J Radiat Oncol 14: 6-9, 2005.

12. Lu H and Yao M: The current status of intensity-modulated radiation therapy in the treatment of nasopharyngeal carcinoma. Cancer Treat Rev 34: 27-36, 2008.

13. Leung TW, Tung SY, Sze WK, et al: Treatment results of 1070 patients with nasopharyngeal carcinoma: an analysis of survival and failure patterns. Head Neck 27: 555-565, 2005.

14. Zhu DG, Qian LT, Zhang HY, et al: Therapeutic effect and prognostic analysis of 164 cases of nasopharyngeal carcinoma after radiotherapy. Acta Univ Medi Anhui 45: 535-538, 2010.

15. Chien CR, Lin HW, Yang CH, et al: High case volume of radiation oncologists is associated with better survival of nasopharyngeal carcinoma patients treated with radiotherapy: a multifactorial cohort analysis. Clin Otolaryngol 36: 558-565, 2011.

16. Liu MT, Hsieh CY, Chang TH, et al: Prognostic factors affecting outcome of nasopharyngeal carcinoma. Jpn J Clin Oncol 33: 501-508, 2003

17. McMillan AS, Pow EH, Kwong DL, et al: Preservation of quality of life after intensity-modulated radiotherapy for early-stage nasopharyngeal carcinoma: results of a prospective longitudinal study. Head Neck 28: 712-722, 2006.

18. Han L, Lin SJ, Pan JJ, et al: Prognostic factors of 305 nasopharyngeal carcinoma patients treated with intensity-modulated radiotherapy. Chin J Cancer 29: 153-158, 2010.

19. Kwong DL, Sham JS, Chua DT, et al: The effect of interruptions and prolonged treatment time in radiotherapy for nasopharyngeal carcinoma. Int J Radiat Oncol Biol Phys 39: 703-710, 1997.

20. O'Meara WP and Lee N: Advances in nasopharyngeal carcinoma. Curr Opin Oncol 17: 225-230, 2005. 